\title{
Comparison of gemcitabine and anthracycline antibiotics in prevention of superficial bladder cancer recurrence
}

\author{
Tian-Wei Wang ${ }^{1}$, Hui Yuan², Wen-Li Diao², Rong Yang ${ }^{2}$, Xiao-Zhi Zhao ${ }^{2}$ and Hong-Qian Guo ${ }^{2^{*}}$ (D)
}

\begin{abstract}
Background: Because of the failure, shortage and related toxicities of Bacillus Calmette-Guérin (BCG), the other intravesical chemotherapy drugs are also widely used in clinical application. Gemcitabine and anthracycline antibiotics (epirubicin and pirarubicin) are widely used as first-line or salvage therapy, but which drug is better is less discussed.

Methods: A total of 124 primary NMIBC patients administered intravesical therapy after transurethral resection of bladder tumor (TURBT) at Nanjing Drum Tower hospital from January 1996 to July 2018. After TURBT, all patients accepted standard intravesical chemotherapy. Recurrence was defined as the occurrence of a new tumor in the bladder. Progression was defined as confirmed tumor invading muscular layer. Treatment failure was defined as need for radical cystectomy $(\mathrm{RC})$, systemic chemotherapy and radiation therapy.

Results: Of the 124 patients who underwent intravesical chemotherapy, 84 patients were given gemcitabine, 40 patients were given epirubicin or pirarubicin, with mean follow-up times (mean \pm SD) of (34.8 \pm 17.9$)$ and (35.9 \pm 22.1$)$ months respectively. The clinical and pathological features of patients show no difference between two groups. Recurrence rate of patients given gemcitabine was $8.33 \%$ (7 out of 84 ), the recurrence rate was $45 \%$ (18 out of 40) for epirubicin or pirarubicin $(P<0.0001)$. The progression rates of gemcitabine, anthracycline antibiotics groups were $2.38 \%$ ( 2 out of 84 ) and $20 \%$ (8 out of 40 ), respectively $(P<0.001$ ). The rate of treatment failure is $8.33 \%$ (7 out of 84 ) and $25 \%$ (10 out of 40$)$, respectively $(P=0.012)$. Gemcitabine intravesical chemotherapy group was significantly related to a lower rate of recurrence $(H R=0.165,95 \% \mathrm{Cl} 0.069-0.397, P=0.000)$, progression $(H R=0.160,95 \% \mathrm{Cl} 0.032-0.799$, $P=0.026)$ and treatment failure $(H R=0.260,95 \% \mathrm{Cl} 0.078-0.867, P=0.028)$.
\end{abstract}

Conclusion: In conclusion, gemcitabine intravesical chemotherapy group was significantly related to a lower rate of recurrence, progression and treatment failure. Gemcitabine could be considered as a choice for these patients who are not suitable for BCG.

Keywords: Gemcitabine, Anthracycline antibiotics, NMIBC, Intravesical therapy

\section{Background}

According to EAU Guidelines, bladder cancer $(\mathrm{BCa})$ is the 11th mostly diagnosed cancer in the population. About $75 \%$ of bladder cancers are NMIBC at initial diagnosis [1], $60 \%$ of these patients experience recurrence and $10 \%$ experience progression in 5 years [2].T1 tumor, HG/G3tumor, CIS, multifocal, recurrent before and tumor size is larger than $3 \mathrm{~cm}$ are regarded as high-risk

\footnotetext{
* Correspondence: dr.ghq@nju.edu.cn

${ }^{2}$ Department of Urology, Nanjing Drum Tower Hospital, Medical School of

Nanjing University, 321 Zhongshan Rd., Nanjing 210008, China

Full list of author information is available at the end of the article
}

tumors. Micropapillary, plasmocytoid, nested, sarcomatoid, microcystic, squamous, and adeno variants of urothelial carcinoma have a poor prognosis [1]. In order to control disease recurrence and progression, intravesical therapy is conventional used after TURBT.

Intravesical BCG therapy is a standard treatment for NMIBC after TURBT [3]. Although BCG has been regarded as the most effective intravesical therapy, it also has disadvantages in clinical use. Firstly, intravesical BCG therapy is associated with adverse effects such as reactive arthritis [4] and Poncet's disease [5]. Secondly, the production of BCG can't meet the market demand,

(C) The Author(s). 2019 Open Access This article is distributed under the terms of the Creative Commons Attribution 4.0 International License (http://creativecommons.org/licenses/by/4.0/), which permits unrestricted use, distribution, and 
which leads to a world-wide shortage of BCG [6]. Thirdly, up to $40 \%$ patients do not respond to intravesical BCG therapy [7]. For all these reasons and more, gemcitabine and anthracycline antibiotics are also widely used clinically as first-line therapy or salvage therapy. However, whether gemcitabine is superior to other intravesical chemotherapy drugs is rarely discussed.

In the present study, we aimed to assess the impact of different intravesical chemotherapy drugs on recurrence, progression and treatment failure in patients with NMIBC.

\section{Methods}

A total of 124 primary NMIBC patients administered intravesical therapy after TURBT at Nanjing Drum Tower hospital from January 1996 to July 2018 were retrospectively analyzed. Inclusion criteria: The patients were primary diagnosed with NMIBC and all of them accepted TURBT followed by intravesical therapy; The demographic, clinical and pathological information was accurate. Histology was affirmed by experienced pathologists at the department of pathology. The grade classification of urothelium carcinomas was according to 2004 WHO classifications and the TNM classification was based on 2002 TNM classification approved by the Union Internationale Contre le Cancer. All patients were stratified according to AUA risk strata. The surgeons evaluated the location, size and numbers of tumors during the operations. All of these patients accepted the immediate chemotherapy after operation. The intravesical therapeutic regimen is shown below: Perfusion once a week for 6 weeks; then once every 2 weeks for 12 weeks; next once a month for 6 months; following once every 2 months until a full year. The respectively per dosage of gemcitabine, pirarubicin and epirubicin is $1000 \mathrm{mg}, 40$ $\mathrm{mg}$ and $40 \mathrm{mg}$. Treatment plan was timely adjusted according to the review results.

\section{Follow up}

Patients were followed up every 3 months with urine cytology and cystoscopy during the first year, every 6 months for the next 2 years, and then yearly thereafter. Ultrasonography, CT scanning, cystoscopy and urinary cytology were used to evaluate recurrence. Recurrence was defined as the occurrence of a new tumor in the bladder. Progression was defined as confirmed tumor invading muscular layer. Treatment failure was defined as need for radical cystectomy $(\mathrm{RC})$, systemic chemotherapy and radiation therapy.

\section{Statistical analysis}

Statistical analysis was performed using SPSS version 22.0. The categorized data was presented as count value, the continuous variables were reported as mean \pm SD. The continuous and categorized data were compared using the $t$ test and Chi square test. Meier method was used to generate the survival curves. The log-rank test was used to verify statistical significance between curves. The multivariable proportional hazards model was used to test prognostic factors with $P<0.2$ in univariate analysis. Multivariate analyses of data were performed using the Cox proportional hazards model. Figures drawing were performing with GraphPad Prism 7. For all statistical comparisons, a value of $p<0.05$ was considered statistically significant.

\section{Results}

Of the 124 patients who underwent intravesical chemotherapy at Nanjing drum tower hospital, 84 patients were given gemcitabine, 40 patients were given epirubicin or pirarubicin, with mean follow-up times (mean $\pm \mathrm{SD}$ ) of $(34.8 \pm 17.9)$ and $(35.9 \pm 22.1)$ months respectively. The clinical and pathological features of patients show no difference between two groups. The baseline characteristics of patients according to treatments are shown in Table 1.

Clinical outcome of the two groups is shown in Table 2. Recurrence rate of patients who was given

Table 1 Characteristics of patients

\begin{tabular}{|c|c|c|c|}
\hline \multirow[t]{2}{*}{ Patient data } & \multicolumn{2}{|c|}{ Intravesical chemotherapy drug } & \multirow[t]{2}{*}{$P$} \\
\hline & $\begin{array}{l}\text { Gemcitabine }(n=84) \\
n(\%)\end{array}$ & $\begin{array}{l}\text { Epirubicin or Pirarubicin } \\
(n=40) \\
n(\%)\end{array}$ & \\
\hline \multicolumn{4}{|l|}{ Gender } \\
\hline Male & $61(72.62 \%)$ & $30(75 \%)$ & \multirow[t]{2}{*}{0.779} \\
\hline Female & $23(27.38 \%)$ & $10(25 \%)$ & \\
\hline \multicolumn{4}{|l|}{ Age (years) } \\
\hline$<65$ & $39(46.43 \%)$ & $14(35 \%)$ & \multirow[t]{2}{*}{0.229} \\
\hline$\geq 65$ & $45(53.57 \%)$ & $26(65 \%)$ & \\
\hline \multicolumn{4}{|l|}{ Multifocality } \\
\hline Single & $41(53.25 \%)$ & $20(57.14 \%)$ & \multirow[t]{2}{*}{0.701} \\
\hline Multiple & $36(46.75 \%)$ & $15(42.86 \%)$ & \\
\hline \multicolumn{4}{|l|}{ Size (cm) } \\
\hline$<3$ & $57(67.86 \%)$ & $25(62.5 \%)$ & \multirow[t]{2}{*}{0.427} \\
\hline$\geq 3$ & $27(32.14 \%)$ & $15(37.5 \%)$ & \\
\hline \multicolumn{4}{|l|}{ Grade } \\
\hline Low & $42(57.53 \%)$ & $19(51.35 \%)$ & \multirow[t]{2}{*}{0.538} \\
\hline High & $31(42.47 \%)$ & $18(48.65 \%)$ & \\
\hline \multicolumn{4}{|l|}{ Risk } \\
\hline Low & $19(22.62 \%)$ & $13(32.50 \%)$ & \multirow[t]{3}{*}{0.452} \\
\hline Immediate & $25(29.76 \%)$ & $9(22.50 \%)$ & \\
\hline High & $40(47.62 \%)$ & $18(45.00 \%)$ & \\
\hline \multicolumn{4}{|l|}{ reTURBT } \\
\hline Yes & $33(39.29 \%)$ & $17(42.50 \%)$ & \multirow[t]{2}{*}{0.733} \\
\hline No & $51(60.71 \%)$ & $23(57.50 \%)$ & \\
\hline \multicolumn{4}{|c|}{ Follow up months } \\
\hline$($ mean $\pm S D)$ & 34.8 (17.9) & 35.9 (22.1) & 0.772 \\
\hline
\end{tabular}


Table 2 Recurrence, progression and treatment failure rates of the two groups

\begin{tabular}{cccc}
\hline & Gemcitabine & Epirubicin or Pirarubicin & $P$ \\
\hline Recurrence & & & \\
Yes & $7(8.33 \%)$ & $18(45 \%)$ & 0.000 \\
No & $77(91.67 \%)$ & $22(55 \%)$ & \\
Progression & & & 0.001 \\
Yes & $2(2.38 \%)$ & $8(20 \%)$ & \\
No & $82(97.62 \%)$ & $32(80 \%)$ & 0.012 \\
Treatment failure & & \\
Yes & $7(8.33 \%)$ & $10(25 \%)$ & \\
No & $77(91.67 \%)$ & $30(75 \%)$ & \\
\hline
\end{tabular}

gemcitabine was $8.33 \%$ (7 out of 84 ), the recurrence rates were $45 \%$ (18 out of 40 ) for epirubicin or pirarubicin $(P<0.001)$. The progression rates of gemcitabine, epirubicin or pirarubicin groups were $2.38 \%$ (2 out of 84 ) and $20 \%$ ( 8 out of 40$)$, respectively $(P<0.01)$. The rate of treatment failure was $8.33 \%$ (7 out of 84 ) and 25\% (10 out of 40$)$, respectively $(P<0.05)$, as shown in Table 2. Taken together, intravesical chemotherapy with different drugs showed an obvious statistically difference, the epirubicin or pirarubicin group had a higher recurrence free survival, progression free survival and treatment failure free survival rate than gemcitabine group.

The log-rank tests show obvious differences between the two groups in recurrence free survival rates, progression free survival rates and treatment failure free survival rates. The Kaplan-Meier estimates of two groups are graphically presented in Fig. 1a-c. Tumor recurrence, progression and treatment failure differ significantly between these groups. Comparing with epirubin or pirarubicin group, gemcitabine group showed obvious advantage in inhibiting tumor recurrence, progression and treatment failure. After univariate analysis, variables with $p<0.2$ were enrolled into multivariate analysis (Table 3 ). Gemcitabine intravesical chemotherapy group was significantly related to a lower rate of recurrence $(\mathrm{HR}=0.165$, 95\% CI 0.069-0.397, $P=0.000$ ), lower rate of progression $(\mathrm{HR}=0.160,95 \%$ CI $0.032-0.799, P=0.026)$ and treatment failure $(\mathrm{HR}=0.260,95 \% \mathrm{CI} 0.078-0.867, P=0.028)$.

\section{Toxicity evaluation}

Common side effects in both groups included chemical cystitis, urinary frequency, hematuria and suprapubic discomfort. Overall $4 \%$ of patients experienced side effects and no patients stopped chemotherapy because of side effects. None of the toxicities were fatal.

\section{Discussion}

Although BCG is used as first-line intravesical therapy for NMIBC patients after TURBT, its shortcomings also poses a management dilemma in clinical application, which force clinicians to search for better therapeutic strategies. As some patients do not respond to BCG or cannot tolerate its side effects, Yang et al. used Gemcitabine and cisplatin (GC) adjuvant chemotherapy instead of BCG intravesical therapy, which showed favorable results [8]. Some researches tried to

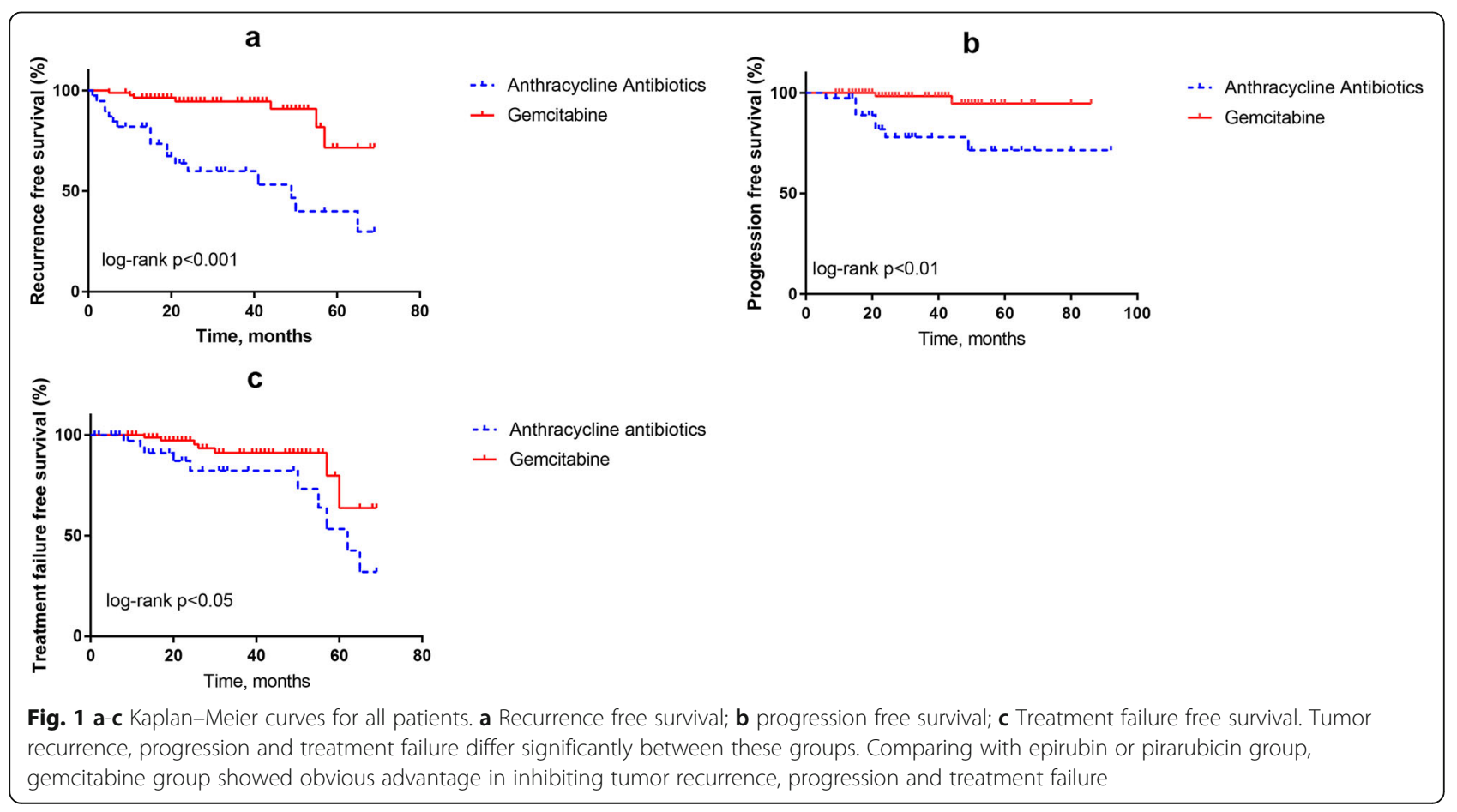


Table 3 Univariate and multivariate standard Cox proportional hazards analysis of variables associated with tumor recurrence, progression and treatment failure in all patients

\begin{tabular}{|c|c|c|c|c|}
\hline Variable & Univariate HR (95\% Cl) & $P$ & Multivariate HR (95\% Cl) & $p$ \\
\hline \multicolumn{5}{|l|}{ Recurrence } \\
\hline Gender & $1.338(0.817-2.191)$ & 0.247 & & \\
\hline Age & $1.497(0.658-3.403)$ & 0.336 & & \\
\hline Multifocality & $1.526(0.642-3.629)$ & 0.339 & & \\
\hline Size & $1.869(0.850-4.108)$ & 0.120 & $2.668(1.146-6.215)$ & 0.023 \\
\hline Grade & $0.443(0.190-1.033)$ & 0.060 & $1.243(0.232-6.664)$ & 0.800 \\
\hline \multicolumn{5}{|l|}{ Risk } \\
\hline \multicolumn{5}{|l|}{ Low $^{a}$} \\
\hline Immediate & $1.934(0.696-5.371)$ & 0.206 & $2.762(0.461-16.543)$ & 0.266 \\
\hline High & $3.019(1.186-7.685)$ & 0.020 & $3.632(0.535-24.633)$ & 0.187 \\
\hline reTURBT & $0.441(0.182-1.068)$ & 0.070 & $0.667(0.134-3.313)$ & 0.620 \\
\hline Gemcitabine vs Epirubicin or Pirarubicin & $0.159(0.066-0.382)$ & 0.000 & $0.179(0.072-0.447)$ & $<0.001$ \\
\hline \multicolumn{5}{|l|}{ Progression } \\
\hline Gender & $1.266(0.583-2.747)$ & 0.552 & & \\
\hline Age & $0.765(0.221-2.644)$ & 0.672 & & \\
\hline Multifocality & $4.063(0.819-20.143)$ & 0.086 & $4.168(0.827-21.000)$ & 0.084 \\
\hline Size & $2.188(0.195-24.519)$ & 0.525 & & \\
\hline Grade & $1.333(0.376-4.727)$ & 0.657 & & \\
\hline \multicolumn{5}{|l|}{ Risk } \\
\hline \multicolumn{5}{|l|}{ Low $^{\mathrm{a}}$} \\
\hline Immediate & $0.993(0.181-5.432)$ & 0.993 & & \\
\hline High & $1.945(0.486-7.780)$ & 0.347 & & \\
\hline reTURBT & $0.323(0.068-1.521)$ & 0.153 & $0.278(0.055-1.394)$ & 0.120 \\
\hline Gemcitabine vs Epirubicin or Pirarubicin & $0.110(0.023-0.518)$ & 0.005 & $0.155(0.031-0.781)$ & 0.024 \\
\hline \multicolumn{5}{|l|}{ Treatment failure } \\
\hline Gender & $0.447(0.128-1.561)$ & 0.207 & & \\
\hline Age & $2.094(0.736-5.960)$ & 0.166 & $2.244(0.591-8.527)$ & 0.235 \\
\hline Multifocality & $2.156(0.739-6.290)$ & 0.160 & $1.447(0.479-4.368)$ & 0.512 \\
\hline Size & $0.897(0.314-2.562)$ & 0.840 & & \\
\hline Grade & $1.485(0.491-4.485)$ & 0.484 & & \\
\hline \multicolumn{5}{|l|}{ Risk } \\
\hline \multicolumn{5}{|l|}{ Low $^{a}$} \\
\hline Immediate & $1.315(0.443-3.906)$ & 0.622 & & \\
\hline High & $0.455(0.099-2.080)$ & 0.310 & & \\
\hline reTURBT & $1.429(0.537-3.804)$ & 0.475 & & \\
\hline Gemcitabine vs Epirubicin or Pirarubicin & $0.377(0.140-1.018)$ & 0.054 & $0.248(0.074-0.830)$ & 0.024 \\
\hline
\end{tabular}

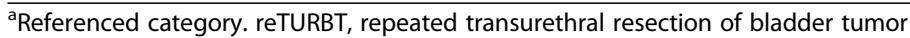

reduce the standard dose of BCG to lessen the shortage of BCG. However, there is no consensus suggesting that intravesical BCG standard dose can be replaced by now [9]. Kyla $\mathrm{N}$. Velaer et al. reported their experience on sequential intravesical gemcitabine and docetaxel as salvage therapy after BCG failure [10]. With the growth demand of BCG and the increasing number of patients, the contradiction will become more and more prominent.
Due to various reasons, gemcitabine is widely used in bladder cancer. In Australia, gemcitabine was setted as firstline intravesical therapy since 2010 [11]. Thiru Prasanna et al. deemed that intravesical gemcitabine had better DFS and lower toxicity when compared with BCG [11]. Pirarubicin and epirubicin has been widely used in intravesical chemotherapy since 1980s and has been proved to be effective. However, William B. Tabayoyong et al. collected seven 
trials about epirubicin and reported that six trials showed no improvement in recurrence with the maintenance treatment to induction [12]. What's more, most researchers think pirarubicin and epirubicin have been only able to reduce recurrence but not progression [13]. Gemcitabine, Pirarubicin and epirubicin are widely used in China, but seldom assessed the efficiency between these therapeutic choices.

In our research, we found a trend toward better recurrence free survival, progression free survival and treatment failure free survival in gemcitabine group. It is also important that gemcitabine intravesical chemotherapy is an independent protective factor not only for recurrence, but also for progression and treatment failure. Through multivariate analysis, we noted that the size of tumor larger than $3 \mathrm{~cm}$ is more likely to recurrent, which is in accord with EAU Guidelines.

There are potential limitations in our analysis. First, it is a retrospective analysis, and our material have limited data. Second, the current study never includes BCG treatment because of limited data. Third, there were other variables never bring into consideration such as molecular subtype of urothelial carcinoma, which may have influenced on results. Most importantly, the limited data may makes the estimate of the treatment effect less robust. Furthermore, a large randomized controlled trial is required to clarify the importance of gemcitabine therapy.

\section{Conclusion}

In conclusion, gemcitabine intravesical chemotherapy group was significantly related to a lower rate of recurrence, progression and treatment failure compared to anthracycline antibiotics group. Gemcitabine is superior to epirubicin or pirarubicin in inhibiting tumor recurrence and progression. We deduce that gemcitabine is also better than epirubicin or pirarubicin in salvage therapy and we will further discuss it. BCG is still the first-line intravesical therapy, but gemcitabine could be considered as a choice for those patients who are not suitable for BCG.

\section{Abbreviations \\ AUA: American urological association; BCa: Bladder cancer; BCG: Bacillus Calmette-Guérin; DFS: Disease free survival; EAU: European Association of Urology; GC: Gemcitabine and cisplatin adjuvant chemotherapy; NMIBC: Non-muscle invasive bladder cancer; RC: Radical cystectomy; TURBT: Transurethral resection of bladder tumor}

\section{Acknowledgements}

The authors thanks to all the staff in the urology department of Nanjing Drum Tower Hospital for their assistance.

\section{Authors' contributions}

W-TW: data collection or management, data analysis and manuscript writing. HY: data collection or management and data analysis. W-LD: data collection or management and data analysis. RY: data collection or management and data analysis. Z-XZ: protocol development. H-QG: protocol development and manuscript editing. All authors have read and approved the final manuscript.

\section{Funding}

Not applicable.

\section{Availability of data and materials}

The datasets used during the current study are available from the corresponding author or first author on reasonable request.

\section{Ethics approval and consent to participate}

The study received the approval from Ethics Committee of Nanjing drum tower hospital (Approval No. 2017023). All procedures performed in studies involving human participants were in accordance with the ethical standards of the institutional research committee and with the 1964 Helsinki declaration and its later amendments or comparable ethical standards. Written informed consent was obtained from all individual participants included in the study.

\section{Consent for publication}

Not applicable.

\section{Competing interests}

The authors declare that they have no competing interests.

\section{Author details}

${ }^{1}$ Nanjing Medical University, 101 Longmian Rd, Nanjing 211166, China. ${ }^{2}$ Department of Urology, Nanjing Drum Tower Hospital, Medical School of Nanjing University, 321 Zhongshan Rd., Nanjing 210008, China.

Received: 2 February 2019 Accepted: 27 September 2019 Published online: 15 October 2019

\section{References}

1. Babjuk M, et al. EAU guidelines on non-muscle-invasive Urothelial carcinoma of the bladder: update 2016. Eur Urol. 2017;71(3):447-61.

2. Ferro $M$, et al. An increased body mass index is associated with a worse prognosis in patients administered BCG immunotherapy for T1 bladder cancer. World J Urol. 2019;37(3):507-14.

3. Kawai K, et al. Bacillus Calmette-Guerin (BCG) immunotherapy for bladder cancer: current understanding and perspectives on engineered BCG vaccine. Cancer Sci. 2013;104(1):22-7.

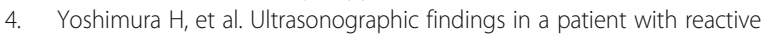
arthritis induced by intravesical BCG therapy for bladder cancer. J Med Ultrason. 2018. https://doi.org/10.1007/s10396-018-0889-7.

5. Sampaio et al. Poncet's disease after the intravesical instillation of Bacillus Calmette - Guérin (BCG):a case report. BMC Res Notes. 2017;10:416.

6. Bandari J, et al. Manufacturing and the market: rationalizing the shortage of Bacillus Calmette-Guérin. Eur Urol Focus. 2018;4(4):481-84.

7. Zlotta AR, Fleshner NE, Jewett MA. The management of BCG failure in nonmuscle-invasive bladder cancer: an update. Can Urol Assoc J. 2009;3(6 Suppl 4):S199-205.

8. Yang GL, et al. Commentary on "A novel treatment strategy for newly diagnosed high-grade $\mathrm{T} 1$ bladder cancer:Gemcitabine and cisplatin adjuvant chemotherapy_A single-institution experience." Urol Oncol. 2017; 35(2):38.e9-38.e15

9. Wu C, et al. Assessing the feasibility of replacing standard-dose Bacillus Calmette-Guérin immunotherapy with other intravesical instillation therapies in bladder cancer patients: a network meta-analysis. Cell Physiol Biochem. 2017:41(4):1298-312.

10. Velaer KN, et al. Experience with sequential intravesical gemcitabine and docetaxel as salvage therapy for non-muscle invasive bladder cancer. Curr Urol Rep. 2016;17(5):38.

11. Prasanna T, et al. Intravesical Gemcitabine versus Intravesical Bacillus Calmette-Guérin for the Treatment of Non-Muscle Invasive Bladder Cancer: An Evaluation of Efficacy and Toxicity. 2017;7(undefined):260.

12. Tabayoyong WB, et al. Systematic review on the utilization of maintenance intravesical chemotherapy in the management of non-muscle-invasive bladder cancer. Eur Urol Focus. 2018;4(4):512-21.

13. Huang $B$, et al. Efficacy of intra-arterial chemotherapy combined with intravesical chemotherapy in T1G3 bladder cancer when compared with intravesical chemotherapy alone after bladder-sparing surgery: a retrospective study. World J Urol. 2019;37(5):823-9.

\section{Publisher's Note}

Springer Nature remains neutral with regard to jurisdictional claims in published maps and institutional affiliations. 